\title{
Low-Drift U-Shaped Thermopile Flow Sensor
}

\author{
M. Dijkstra, T.S.J. Lammerink, M.J. de Boer, J.W. Berenschot, R.J. Wiegerink, M.C. Elwenspoek \\ $\mathrm{MESA}^{+}$Institute for Nanotechnology \\ University of Twente \\ Enschede, The Netherlands \\ m.a.dijkstra@ewi.utwente.nl
}

\begin{abstract}
A thermal flow sensor has been realised consisting of a freely-suspended U-shaped microchannel. The structure is symmetrically heated by a heater at the top of the $U$-shape. The thermal imbalance caused by liquid flow is sensed by an integrated $\mathrm{Al} /$ poly-Si $^{++}$thermopile. The $\mathrm{U}$-shape microchannel facilitates the integration of a large number of thermocouple junctions, resulting in a highly-sensitive calorimetric flow sensor (40 $\mathrm{mV} / \mu \mathrm{l} \cdot \mathrm{min}^{-1}$ at $2 \mathrm{~mW}$ heating power). The heating power is controlled accurately by forcing a current, while measuring the voltage over the heater resistor. Influences of thermal gradients across the chip are minimised by the freelysuspended microchannel ends being fixed to the substrate over a small distance. The inherently zero-offset of the thermopile can furthermore be exploited in a control system cancelling temperature imbalance by liquid flow using additional heaters. This makes the flow sensor independent of heater resistor values and thermopile output characteristics. Accurate measurements up to $400 \mathrm{nl} \cdot \mathrm{min}^{-1}$ water flow have been obtained applying a temperature-balancing control system.
\end{abstract}

\section{INTRODUCTION}

The miniaturisation of micro-fluidic components in chemical analysis, synthesis, biotech and nanotechnology asks for the accurate and reliable measurement of tiny fluid flow rates down to a few $\mathrm{nl} \cdot \mathrm{min}^{-1}[1,2]$. The accuracy of these micro-machined thermal flow sensors is ultimately influenced by drift in transducer materials, for instance the continuing drift in resistance values of thin-film metal layers used. Ambient thermal effects can have influence on the accuracy as well. The research presented in this paper aims at developing sensor structures and operation principles [3-5] that are independent of actual heater resistance values and thus do not suffer from resistance drift. So far, straight microchannels have been used, applying thermopile read-out and power feedback [6], cancelling drift in the thermopile output voltage and heater resistors. Although a significant improvement in drift performance was obtained, these devices remain sensitive for thermal gradients over the chip. In this paper we present an improved sensor design facilitating the integration of a large number of thermocouple junctions and eliminating the dependency on thermal gradients over the chip, because both ends of the U-shaped microchannel are fixed to the substrate over a small distance.

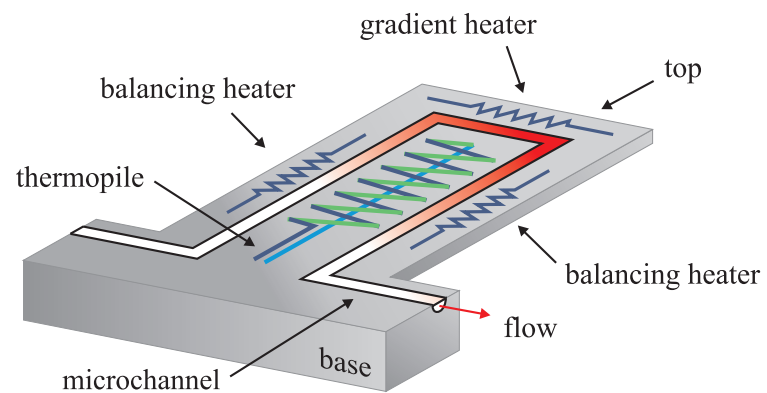

Figure 1. Schematic U-shaped microchannel flow sensor structure, with integrated heaters and thermopile.

Fig. 1 shows a representation of the freely-suspended Ushaped thermal flow sensor. Liquid flows through a microchannel embedded in the substrate. The freelysuspended U-shape microchannel is heated at the top by the gradient heater $R_{H}$, creating a thermal gradient towards the base of the freely-suspended structure. Without flow $Q$ both sides of the U-shape are heated symmetrically. When flow is applied, one side of the U-shape decreases in temperature, while the other side increases in temperature (Fig. 2). A thermopile with junctions on either side of the U-shape measures the temperature difference and gives an output voltage $V_{T C}$ corresponding to the temperature imbalance. In this way, the sensor can be used as a calorimetric thermal flow sensor, with the voltage output of the thermopile giving a measure of the flow rate, while the power in the gradient heater can be controlled accurately using 4-point measurement.

Additionally a control system can be used to exploit the zero-offset differential temperature-dependent output $V_{T C}$ of the thermopile, providing for an inherently drift-free zerooffset error signal (Fig. 3), in order to obtain a flow sensor that is independent of resistance drift and non-linear thermopile characteristics $m_{T C}\left(\Delta T_{T C}\right)$. This requires the cancellation of the thermopile output voltage and thus the temperature imbalance between both sides of the U-shape, requiring two additional balancing heater resistors $R_{1}, R_{2}$. The power dissipated in both balancing heaters is also 


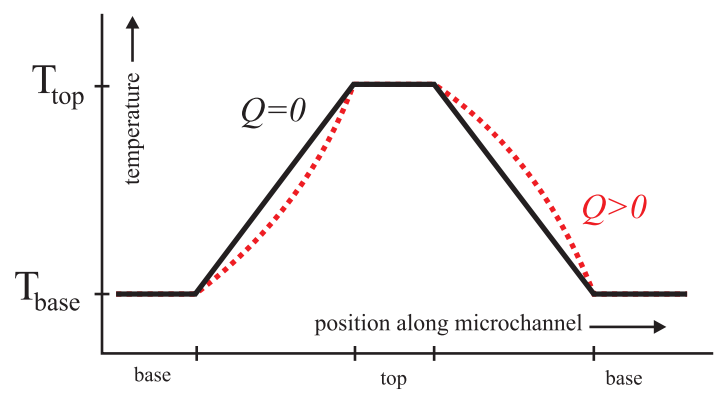

Figure 2. Temperature profiles along the microchannel.

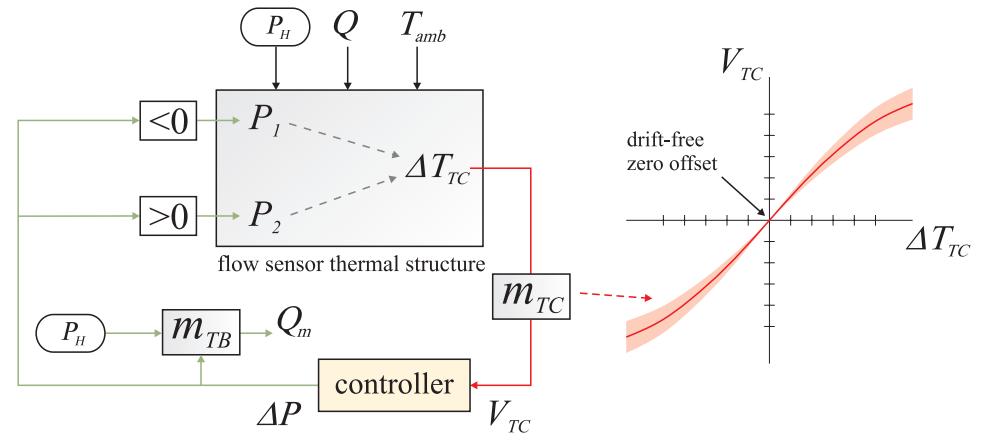

Figure 3. Zero-offset thermopile based power-feedback control system. accurately controlled using 4-point measurement. The output voltage $V_{T C}$ of the thermopile is cancelled by controlling a power difference $\Delta P$ between the two heater resistors, while dissipating a constant power $P_{H}$ in the gradient heater, according to the temperature-balancing anemometry principle [3]. The power difference $\Delta P$ is dissipated in one of the balancing heater resistors, with $R_{I}$ dissipating power when $\Delta P$ is negative and $R_{2}$ dissipating power when $\Delta P$ is positive, depending on the polarity of the thermopile. A $\mathrm{PI}$-controller can be used to minimise the error $V_{T C}$, however a linear fit can be made, directly obtaining the correct $\Delta P$, with two measurement points near $\Delta P$. This means the controller does not have parameters to adjust, cannot become instable and does not suffer from settling time. The temperature distribution changes on applying a fluid flow due to forced convection. This results in the relation $\Delta P / P_{H}$ being dependent on the flow rate $Q$. A measure of the flow rate $m_{T B}\left(\Delta P / P_{H}\right)$ can be obtained, with $\Delta P / P_{H}$ being linear for small flow rates.

\section{SENSOR FABRICATION}

The fabricated thermopile flow sensor incorporates mechanically strong freely-suspended surface microchannels for on-chip transport of fluid. Surface microchannels are fabricated without the requirement for a sacrificial layer and allow for the integration of sensor elements in close proximity to the fluid [7].

Fig. 4 gives a schematic overview of the process scheme for the fabrication of the thermopile flow sensor. Surface microchannels are created by isotropic dry etching, using high-density $\mathrm{SF}_{6}$ plasma with zero self-bias, through etch holes $2 \mu \mathrm{m}$ in width, in a low-stress $500 \mathrm{~nm}$ silicon-rich silicon-nitride ( $\mathrm{SiRN})$ layer. The etch holes and inner surfaces of the microchannels are conformally coated by a second low-stress LPCVD deposited $1.2 \mu \mathrm{m}$ SiRN layer, resulting in completely sealed microchannels, while leaving a planar substrate surface for the integration of an $\mathrm{Al} /$ poly- $^{+{ }^{++}}$thermopile and $\mathrm{Al}$ heater resistors. The thermopile is created by LPCVD deposition and boron doping by solid source diffusion of a $200 \mathrm{~nm}$ poly-Si ${ }^{++}$layer and sputtering of a $200 \mathrm{~nm} \mathrm{Al} \mathrm{layer} \mathrm{(Fig.} \mathrm{4a).} \mathrm{The} \mathrm{surface}$ microchannels are released by $\mathrm{SF}_{6}$ plasma etching, for thermal isolation from the heat-conducting substrate, with the photoresist mask protecting the sensor elements during the release (Fig. 4b). The photoresist is removed after fluidic entrance holes are etched through the SiRN layer, allowing for direct interfacing to the microchannels on the substrate surface.

Fig. 5a shows micrographs of the fabricated thermopile flow sensor. The sensor contains five parallel $15 \mu \mathrm{m}$ diameter microchannels, forming a $1.5 \mathrm{~mm}$ long U-shape freely-suspended over a thermal isolation cavity etched in the silicon substrate. The thermal isolation cavity has a depth of $230 \mu \mathrm{m}$ outside the U-shape, while the etch depth inside the U-shape is only $130 \mu \mathrm{m}$, due to the RIE lag around the thermocouple structures. The parallel microchannels have $200 \mu \mathrm{m}$ total width, which allows for the integration of the $\mathrm{Al}$ heater resistors and $\mathrm{Al} /$ poly-Si $^{++}$thermocouple junctions. The thermopile has 34 junctions on either side of the U-shape structure. The Al heater resistors have four-point measurement contacts for accurate resistance measurements. Fig. 5b shows a cross-section through a microchannel, having a planar wafer surface, on which the sensor structures are fabricated.

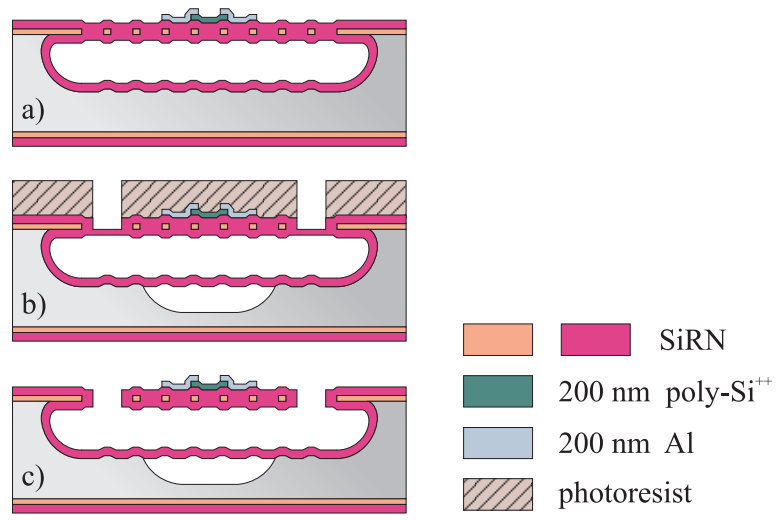

Figure 4. Process scheme for the fabrication of the microchannel thermopile U-shaped microchannel flow sensor. 


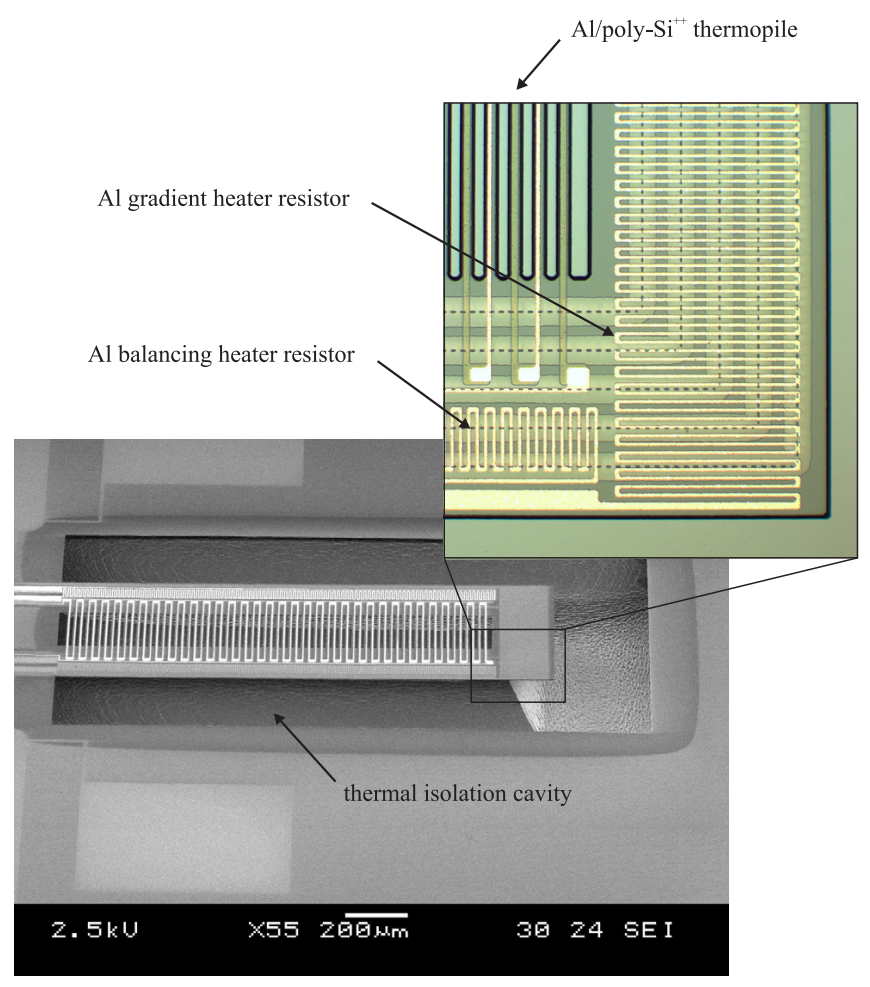

a)

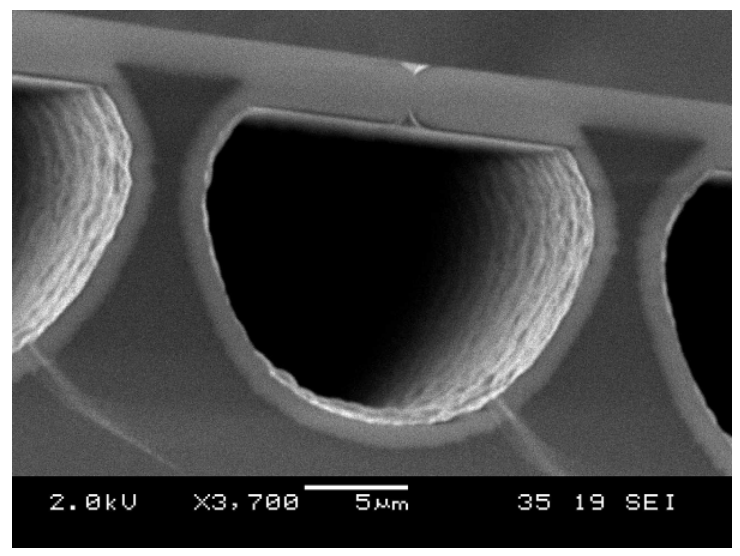

b)

Figure 5. a) U-shaped microchannel flow sensor. The inset shows the $\mathrm{Al}$ heater resistors and $\mathrm{Al} /$ poly-Si ${ }^{++}$thermocouples. b) Cross-section of a microchannel, with a planar surface for the integration of sensor structures.

\section{EXPERIMENTAL RESULTS}

Fabricated $12.5 \times 12.5 \mathrm{~mm}$ thermopile flow sensor chips were measured using a chip holder for electrical and fluidic connections (Fig. 6). The chip-holder contains O-rings for fluidic interfacing and twelve pogo-pins for electrical connection to the sensor chip. Water flow was applied by an elevation head $\Delta h$ up to a few meters, giving a stable flow rate. The flow is restricted by the hydraulic resistance of the microchannels and was determined by microbalance weighing.

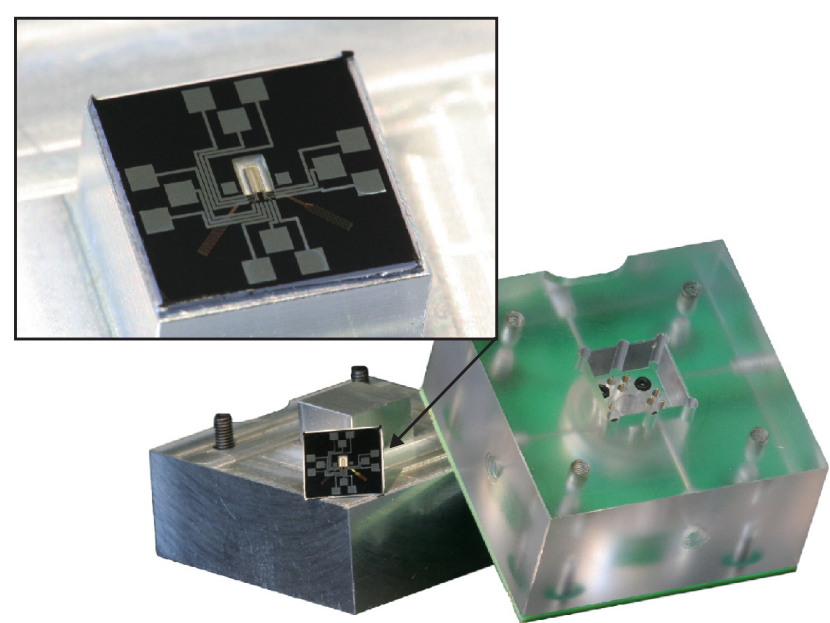

Figure 6. Chip holder for fluidic and electrical interfacing with the sensor chip, measuring $12.5 \times 12.5 \mathrm{~mm}$. The inset shows an overview of the U-shaped microchannel flow sensor chip.

A source meter (Keithley 2420) was used to control the power in the gradient heater resistor. A two-channel source meter (Keithley 2602) was used to control the power on the balancing heater resistors. The thermopile output voltage was measured by a nano-volt meter (HP 34420A). The measurements were performed using MATLAB.

The flow sensor was characterised as an open loop calorimetric flow sensor applying different gradient heating powers $P_{H}$. The flow sensor shows a linear output response up to $500 \mathrm{nl} \cdot \mathrm{min}^{-1}$ water flow rate (Fig. 7), with maximum sensitivity $\left(40 \mathrm{mV} / \mu \mathrm{l} \cdot \mathrm{min}^{-1}\right)$ at $2 \mathrm{~mW}$ heating power. At zero flow rate the thermal gradient is uniform along the U-shape structure, which gives no thermopile voltage and hence no output signal.

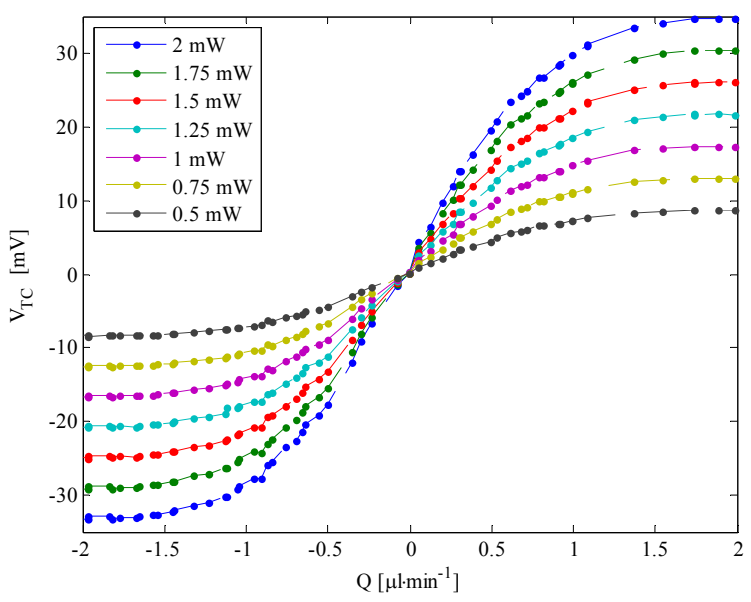

Figure 7. Caloremetric sensor output response $V_{T C}$ versus water flow rate $Q$ with different heating powers $P_{H}$ applied. 


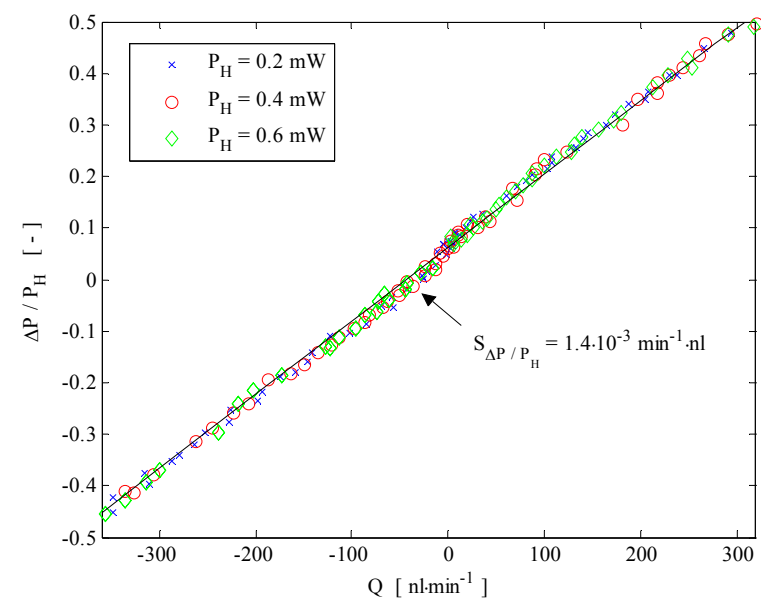

Figure 8. Measured sensor output response $\Delta P / P_{H}$ as function of water flow rate $Q$ at various gradient heating powers $P_{H}$.

The flow sensor was additionally characterised applying temperature balancing, by dissipating power in the balancing heater resistors, thus cancelling the temperature imbalance due to water flow. Fig. 8 shows the measured $\Delta P / P_{H}$ sensor output having a linear response independent of gradient heating power $P_{H}$ with $1.4 \cdot 10^{-3} \mathrm{~min} \cdot \mathrm{nl}^{-1}$ sensitivity.

Fig. 9 shows the measured control system response on applying a changing water flow rate, with $0.4 \mathrm{~mW}$ gradient heating power. The sensor output $\Delta P / P_{H}$ gives a correct measure of the flow rate, while being independent of heater resistance values. Fig. 9 shows that the balancing power $\Delta P$ is being dissipated in $R_{l}$ instead of $R_{2}$ after $\Delta P$ changes sign, while the thermopile voltage is correctly regulated to zero.

\section{CONCLUSIONS}

A thermal flow sensor consisting of a freely-suspended U-shape microchannel with integrated $\mathrm{Al}$ heaters and an $\mathrm{Al} /$ poly- $\mathrm{Si}^{++}$thermopile has successfully been fabricated. A linear calorimetric sensor output was obtained for water flow rates up to $500 \mathrm{nl} \cdot \mathrm{min}^{-1}$, with $40 \mathrm{mV} / \mu \mathrm{l} \cdot \mathrm{min}^{-1}$ sensitivity at $2 \mathrm{~mW}$ heating power. The heating power dissipated has been made independent of the heater resistance value, by using power control applying 4-point measurement on the gradient heater resistor. Influence of thermal gradients across the chip has been minimised by the U-shape microchannel design. The dependency on the non-linear characteristics of the thermopile has been minimised by using a control system, cancelling the temperature imbalance across the thermopile. A linear sensor response for water flow rates up to $300 \mathrm{nl} \cdot \mathrm{min}^{-1}$, with $1.4 \cdot 10^{-3} \mathrm{~min} \cdot \mathrm{nl}^{-1}$ sensitivity, was measured using temperature balancing. The temperature-balancing control system operates correctly, without the possibility of instability and without settling time.
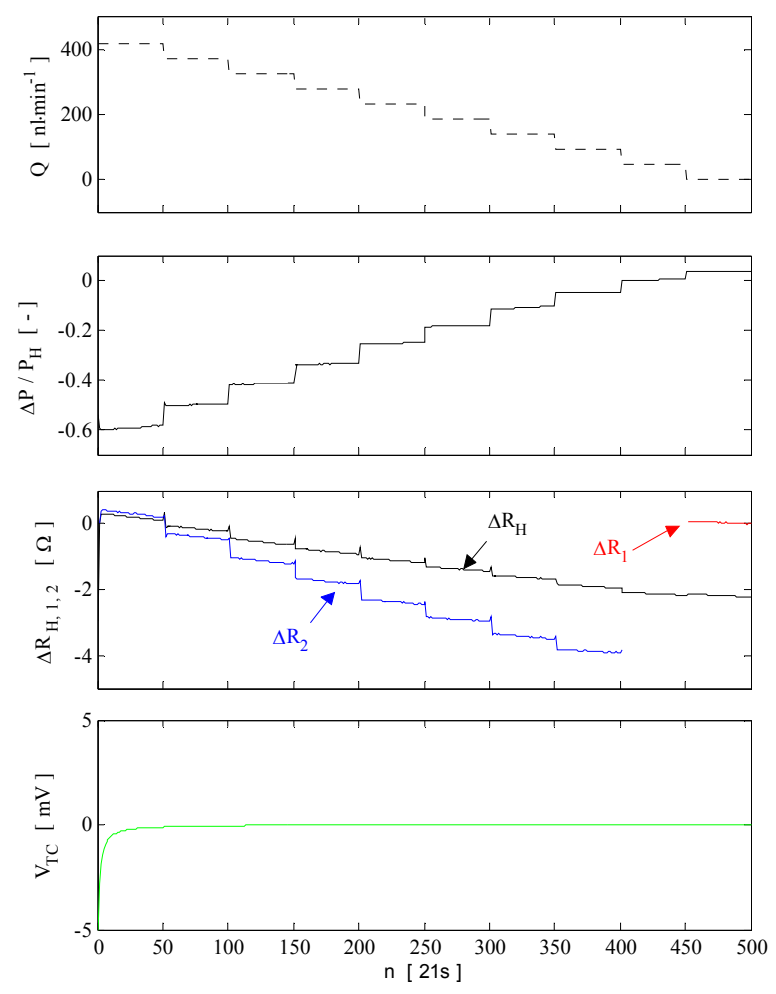

Figure 9. Measured sensor control system response at changing water flow rate. The gradient heating power $P_{H}$ is kept constant at $0.4 \mathrm{~mW}$.

\section{REFERENCES}

[1] M. Dijkstra, M.J. de Boer, J.W. Berenschot, T.S.J. Lammerink, R.J. Wiegerink, M. Elwenspoek, "Miniaturized Thermal Flow Sensor with Planar Integrated Sensor Structures on Semicircular Surface Channels", Sensors and Actuators A., Vol. 143, 2008

[2] Y. Mizuno, M. Liger, Y-C. Tai, "Nanofluidic Flowmeter Using Carbon Sensing Element", Proc. of IEEE MEMS, 2004

[3] T.S.J. Lammerink, N.R. Tas, G.J.M. Krijnen, M. Elwenspoek, “A New Class of Thermal Flow Sensors Using $\Delta \mathrm{T}=0$ as a Control Signal", Proc. IEEE MEMS, 2000

[4] P. Bruschi, A. Diligente, D. Navarrini, M. Piotto, "A Double Heater Integrated Gas Flow Sensor with Thermal Feedback", Sensors and Actuators A., Vol. 123-124, 2005

[5] M. Domínguez, F.N. Masana, V. Jiménez, S. Bermejo, J. Amirola, J. Ballester, N. Fueyo, L.M. Castañer, "Low-Cost Thermal $\Sigma-\Delta$ Air Flow Sensor", J. IEEE Sensors, Vol. 2, 2002

[6] M. Dijkstra, T.S.J. Lammerink, M.J. de Boer, J.W. Berenschot, R.J. Wiegerink, M. Elwenspoek, "Low-Drift Flow Sensor with ZeroOffset Thermopile-Based Power Feedback", Proc. of DTIP, 2008

[7] M. Dijkstra, M.J. de Boer, J.W. Berenschot, T.S.J. Lammerink, R.J. Wiegerink, M. Elwenspoek, "A Versatile Surface Channel Concept for Microfluidic Applications", J. Micromechanics and Microengineering, Vol. 17, 2007 